\title{
artigo
}

\section{Prevalência de doenças crônicas não transmissíveis em idosos longevos em um município no interior do Amazonas}

Prevalence of chronic non-communicable diseases in long-term elderly people in a municipality inside Amazonas Prevalencia de enfermedades crónicas no transmisibles en personas mayores de larga duración en un municipio dentro de las Amazonas

\begin{abstract}
RESUMO
Objetivo: Identificar a prevalência de doenças crônicas não transmissíveis (DCNT) autorreferidas em idosos longevos e traçar seu perfil sociodemográfico. Método: Estudo descritivo, transversal e de abordagem quantitativa, realizado com 135 idosos longevos, de ambos os sexos, cadastrados em 12 Unidades Básicas de Saúde do município de Coari, interior do Amazonas, Brasil. A coleta das informações ocorreu por meio de formulário estruturado, no domicílio do idoso, no período de dezembro de 2019 a fevereiro de 2020. Os dados foram analisados com o auxílio do software IBM SPSS Statistics. Resultados: Dentre os 135 idosos, a média de idade foi de 85,8 ( $d p= \pm 5)$, predominância do sexo feminino $(61,5 \%)$, com baixa escolaridade $(56,3 \%$ ). A prevalência de DCNT na população estudada foi de $85,9 \%$ idosos, sendo a hipertensão arterial sistêmica (HAS) a comorbidade mais frequente em ambos os sexos. Conclusão: Constatou-se a presença de doenças crônicas, com prevalência de HAS, em ambos os sexos.

DESCRITORES: Idoso de 80 Anos ou Mais. Doenças Crônicas. Atenção Primária de Saúde.
\end{abstract}

\begin{abstract}
Objective: To identify the prevalence of self-reported chronic non-communicable diseases (NCDs) in long-lived elderly, and outline their sociodemographic profile. Method: A descriptive, cross-sectional study with a quantitative approach, carried out with 135 long-lived elderly people, of both sexes, registered in 12 Basic Health Units in the municipality of Coari, in the interior of Amazonas, Brazil. The information was collected using a structured form, at the elderly's home, from December 2019 to February 2020. The data were analyzed using the IBM SPSS Statistics software. Results: Among the 135 elderly, the average age was $85.8(S D= \pm 5)$, predominantly female $(61.5 \%)$, with low education (56.3\%). The prevalence of NCDs in the studied population was $85.9 \%$ elderly, with systemic arterial hypertension (SAH) being the most frequent comorbidity in both sexes. Conclusion: The presence of chronic diseases, with prevalence of SAH, was found in both sexes.

DESCRIPTORS: Elderly 80 Years or Older. Chronic diseases. Primary Health Care.
\end{abstract}

\section{RESUMEN}

Objetivo: Identificar la prevalencia de enfermedades crónicas no transmisibles (ENT) auto notificadas en ancianos longevos y delinear su perfil sociodemográfico. Método: Estudio descriptivo, transversal con abordaje cuantitativo, realizado con 135 ancianos longevos, de ambos sexos, registrados en 12 Unidades Básicas de Salud del municipio de Coari, en el interior de Amazonas, Brasil. La información se recopiló mediante un formulario estructurado, en el domicilio de la tercera edad, desde diciembre de 2019 hasta febrero de 2020. Los datos se analizaron mediante el software IBM SPSS Statistics. Resultados: Entre los 135 ancianos, la edad promedio fue de $85,8(\mathrm{DE}= \pm 5)$, predominantemente mujeres $(61,5 \%)$, con baja escolaridad $(56,3 \%)$. La prevalencia de ENT en la población estudiada fue del 85,9\% ancianos, siendo la hipertensión arterial sistémica (HAS) la comorbilidad más frecuente en ambos sexos. Conclusión: La presencia de enfermedades crónicas, con prevalencia de HAS, se encontró en ambos sexos.

DESCRIPTORES: Anciano de 80 años o más. Enfermedades crónicas. Atención Primaria de Salud.

RECEBIDO EM: 16/12/2020 APROVADO EM: 11/01/2021

\section{Tatiana Caroline Lima Lobato}

Acadêmica de Enfermagem. Discente da Universidade Federal do Amazonas.

ORCID: 0000-0002-4031-8027 


\section{Deyvylan Araujo Reis}

Doutor, Enfermeiro. Docente da Universidade Federal do Amazonas.

ORCID: 0000-0001-9314-3745

\section{Jaynne de Souza Dantas}

Acadêmica de Enfermagem. Discente da Universidade Federal do Amazonas.

ORCID: 0000-0003-2989-7029

\section{Dhienifã Brena Marinho de Souza}

Acadêmica de Enfermagem. Discente da Universidade Federal do Amazonas. ORCID: 0000-0001-9342-8628

\section{Adria Dantas de Souza}

Acadêmica de Enfermagem. Discente da Universidade Federal do Amazonas. ORCID: 0000-0001-6747-1972

\section{Gabriel Garcia Siqueira}

Acadêmico de Enfermagem. Discente da Universidade Federal do Amazonas. ORCID: 0000-0003-2892-2595

\section{INTRODUÇÃO}

0 processo de envelhecimento com as alterações que acarreta no corpo de cada indivíduo deve ser entendido e apreciado como uma parte do processo natural do desenvolvimento humano. Esse processo é inerente a todo indivíduo, e todos os dia cada um de nós envelhece um pouco mais, desse modo, é crucial que ao longo desse processo se tome atitudes saudáveis para a que possam influenciar em uma melhor qualidade de vida na velhice ${ }^{1}$.

A presença de uma escassa procura aos serviços de saúde nos estágios iniciais de uma doença e uma baixa adesão ao seu tratamento, geralmente estão associadas a países que possuem uma menor estrutura socioeconômica e o pouco nível de escolaridade $^{2}$. Na população idosa especificamente, a prevalência de Doença Cônica Não Transmissível (DCNT) é comumente observada como fator relevante para o desenvolvimento de incapacidade física, principalmente quando não tratada de forma adequada ${ }^{3}$.

Dentre as DCNT mais prevalentes em idosos as doenças cardiovasculares, o câncer, as doenças respiratórias crônicas e diabetes mellitus tem grande destaque. Essas doenças estão muitas vezes relacionadas a fatores de risco como: tabagismo, álcool, histórico familiar, sedentarismo, alimentação não saldável e obesidade ${ }^{2,4}$. A depressão também tem se tornado uma enfermidade cada vez mais comum entre os idosos, especialmente as do sexo feminino, estando geralmente a associadas a comorbidades cardiovasculares e as cerebrovasculares 5 .

\section{O processo de}

envelhecimento com

as alterações que

acarreta no corpo

de cada indivíduo

deve ser entendido

e apreciado como

uma parte do

processo natural do

desenvolvimento

humano.
Em revisão, Melo et al. ${ }^{6}$ apontam uma frequência crescente de múltiplas doenças crônicas em idosos, realçando que essa realidade vem se tornando uma condição bastante comum, chegando a variar de $30,7 \%$ a $57,0 \%$ dos casos de DCNT em idosos, sendo habitualmente influenciada por fatores socioeconômicos, demográficos, estilo de vida e estrutura familiar. Concomitantemente os idosos em condição de multimorbidade tendem a passar por um maior número de hospitalizações, o que pode influenciar em uma autopercepção de saúde negativa, além da necessidade do consumo de uma variedade de medicamentos que consequentemente podem aumentar o desenvolvimento de efeitos adversos?

Dentro do modelo preventivo, a atenção primária à saúde funciona como instrumento essencial não só para a prevenção das doenças crônicas, mas também manutenção da capacidade funcional, visto que ela possui o domínio sobre as estratégias mais eficazes na prevenção de agravos a saúde dos idosos que possam levar à perda da capacidade funcional ${ }^{3}$. Dessa forma, enfatiza-se a necessidade de intervenções de outras áreas, não somente a de saúde, no desenvolvimento de ações e políticas completas para melhoria no processo de cuidar do idoso, pois a relação deste com os serviços de saúde é complexa e capaz de refletir problemáticas que impactam 


\section{artigo}

Lobato, T.C.L.; Reis, D.A.; Dantas, J.S.; Souza, D.B.M.; Souza, A.D.; Siqueira, G.G.;

Prevalência de doenças crônicas não transmissiveis em idosos longevos em um município no interior do Amazonas

negativamente na qualidade de vida dessa população e de toda a sociedade ${ }^{8}$.

A relevância da temática do presente estudo, provem de possibilitar identificar a prevalência de DCNT para a construção e caracterização de um perfil dos idosos que sofrem com essas comorbidades, e ainda possibilitar subsídios para a formulação políticas de saúde voltadas para a prevenção desses agravos, e assim incentivar as unidades básicas de saúde (UBS) a realizarem atividades que informem e sensibilizem a população quanto aos fatores de risco e a importância de preveni-los o mais precocemente possível, visando à redução de comorbidades ${ }^{4}$.

O presente estudo teve como objetivo identificar a prevalência de DCNT autorreferida do idoso longevo do município de Coari no Estado do Amazonas. Diante disso, emerge a seguinte questionamento: Qual é a prevalência de doença crônica não transmissível em idosos longevos?

\section{MÉTODO}

Trata-se de um estudo descritivo, com delineamento transversal com abordagem quantitativa. Esta pesquisa integra um projeto maior intitulado "Saúde do idoso longevo: capacidade funcional e doença crônica não transmissível", realizado na zona urbana de um município do interior do Amazonas, Brasil.

O local de estudo é um município da região do Médio Solimões chamada de Coari no Estado do Amazonas, com a população estimada em 75.965 habitantes do último censo demográfico do ano de $2010^{9}$.

A seleção dos participantes para o presente estudo partiu dos dados da Secretária de Saúde do município de Coari que disponibilizou o número de idosos igual ou superior a 80 anos cadastrados em todas as Unidades Básica de Saúde (UBS), que correspondeu a 557 indivíduos. A partir desses dados, foi realizado o cálculo amostral pela fórmula destinada para população infinita, em que inicialmente obteve 228 idosos para amostra. Considerou-se o nível de confiança de $95 \%$ e margem de erro de $0,05 \%$. Na busca dos dados documentais disponibilizados por cada UBS, constatou-se que eram acompanhados 227 idosos longevos da sua área de abrangência das 12 UBS e que existiam 42 óbitos, resultando no total de 185 . Desse modo a amostra final correspondeu a 135 idosos longevos e 50 perdas (recusa na participação no estudo, idosos não encontrados no domicílio, mudanças de domicílio ou na cidade).

A coleta foi realizada no domicílio de cada participante, e ocorreu no período de dezembro de 2019 a março de 2020, no município de Coari no Estado do Amazonas. Como critério de elegibilidade foram incluídos os indivíduos com idade igual ou superior de 80 anos, de ambos os sexos, cadastrados na Unidade Básica de Saúde do município. Foram excluídos os indivíduos não cadastrados nas UBS, indígenas, idosos sem condições clínicas e cognitiva para compreender as perguntas dos instrumentos de coleta de dado.

Os participantes incluídos no estudo foram visitados em suas residências, onde foram devidamente informados sobre a pesquisa, confirmando sua participação por meio da assinatura do termo de consentimento livre e esclarecido, e das informações fornecidas aos pesquisadores através de formulário estruturado. Este instrumento abordou informações de interesse para o estudo, tais como: variáveis demográficas (idade e sexo), socioeconômica (situação conjugal, escolaridade, renda pessoal e familiar, religião, ocupação atual), domicílio (quanto pessoas no domicílio, quem mora), condição de saúde (comorbidades). Posteriormente os dados coletados foram analisados e apresentados utilizando as técnicas usuais empregadas em estudo descritivo, para variáveis categóricas, a partir de frequência absolutas e percentuais; e média, desvio padrão, mínimo e máximo para os numéricos. Para a associação foi aplicado o Teste Exato de Fisher e Teste Qui-quadrado.

$\mathrm{O}$ referido projeto tem aprovação no Comitê de Ética em Pesquisa (CEP) da Universidade Federal do Amazonas (UFAM) $n^{\circ}$ CAAE 20056119.0.0000.5020. Está investigação encontrar-se em conformidade com a resolução 466/12 do Conselho Nacional de Saúde.

\section{RESULTADOS E DISCUSSÃO}

Participaram do estudo 135 idosos longevos, com média de idade de 85,8 $(\mathrm{dp}= \pm 5)$, com o mínimo de 80 máxima de 102 anos. Destes, a faixa etária predominante $(79,3 \%)$ variou entre 80 e 89 anos, sendo a maioria dos idosos (61,5\%) do sexo feminino, conforme os dados apresentados na Tabela 1.

Os resultados são semelhantes aos estu-

Tabela 1. Caracterização de variáveis demográfica e socioeconômicas dos idosos longevos (N=135). Coari, AM, 2020.

VARIÁVEIS

$N(\%)$

MÉDIA (DESVIO-PADRÃO)

Faixa etária

80-89

$107(79,3)$
90-99

$26(19,3)$

$\geq 100$

$2(1,4)$

Sexo

Feminino

$83(61,5)$

Masculino

$52(38,5)$

Escolaridade*

0

$76(56,3)$

1-4

$34(25,2)$

$1,9( \pm 2,5)$

$85,8( \pm 5,0)$

$\geq 5$

$25(18,5)$

Religião 


$\begin{array}{lc}\text { Católico } & 94(69,6) \\ \text { Protestante } & 39(28,9) \\ \text { Sem religião } & 2(1,5) \\ \text { Situação conjugal } & 22(16,3) \\ \text { Solteiro (a) } & 42(31,1) \\ \text { Casado (a) } & 71(52,6) \\ \text { Viúvo (a) } & 112(83,0) \\ \text { Renda Individual** } & 23(17,0) \\ 0-2 & 118(87,4) \\ \text { >2 } & 16(11,9) \\ \text { Renda familiar** } & 1(0,7) \\ 1-2 & 1,2( \pm 0,4) \\ 3-4 & 1,8( \pm 0,8) \\ \geq 5 & \end{array}$

dos realizados como os idosos em cidades do interior do Rio Grande do Sul e na capital do Pará, os quais ressaltaram o predomínio do gênero feminino entre participantes, corroborando com o achado da maioria dos estudos nacionais ${ }^{10-13}$ e internacionais ${ }^{14,15}$. Com relação a esse aspecto, deve ressaltar que esse fenômeno da feminização da velhice, evidente em uma escala global, geralmente está relacionado a alta prevalência de mortalidade entre o sexo masculino e uma visível maior preocupação entre as mulheres com o autocuidado

em saúde, o que pode explicar a maior longevidade gênero feminino ${ }^{16}$.

A baixa escolaridade e a renda entre os participantes representaram resultados expressivos, no qual, o analfabetismo $(56,3 \%)$ chegou a estar presente em mais da metade dos casos, em que pelo menos $87,4 \%$ possuem uma renda familiar de um a dois salários-mínimos. Levando-se em consideração que $82,2 \%$ desses idosos relataram residir com 1 a 5 pessoas, e apresentando uma baixa renda per capita familiar. Estes resultados ainda que discretamente

Quadro 1. Distribuição de morbidade autorreferida com relação ao sexo dos idosos longevos ( $N=135)$. Coari, AM, 2020.

\begin{tabular}{|l|c|c|}
\hline \multirow{2}{*}{ MORBIDADE } & FEMININO & MASCULINO \\
\cline { 2 - 3 } & $\mathbf{N}(\%)$ & $\mathbf{N}(\%)$ \\
\hline Hipertensão Arterial Sistêmica & $60(72,3)$ & $34(65,4)$ \\
\hline Dorsalgia & $23(27,7)$ & $18(34,6)$ \\
\hline Artrite reumatoide & $19(22,9)$ & $13(25,0)$ \\
\hline Diabetes Mellitus & $18(21,7)$ & $6(11,5)$ \\
\hline Gastrite Crônica & $14(16,9)$ & $5(9,6)$ \\
\hline Osteoporose & $15(18,1)$ & $2(3,8)$ \\
\hline Demência & $10(12,0)$ & $6(11,5)$ \\
\hline Alzheimer & $3(3,6)$ & $1(1,9)$ \\
\hline Insuficiência cardíaca congestiva & $1(1,2)$ & $3(5,8)$ \\
\hline Doença de Parkinson & $1(1,2)$ & $2(3,8)$ \\
\hline *Outras & $3(3,2)$ & $2(3,8)$ \\
\hline *Outras: neoplasia, doença renal crônica, asma etranstorno depressivo. & \\
\hline
\end{tabular}

corroboram com uma outra análise realizada em Campina Grande-PB, cujos dados concernentes à escolaridade revelam que $39,2 \%$ idosos pesquisados apresenta baixa escolaridade, entre 1 a 4 anos de estudo. Já com relação ao aspecto socioeconômico, o mesmo estudo mostrou que pelo menos 3/4 dos idosos recebem apenas um salário mínimo ${ }^{16}$.

Importa registrar que se deve considerar a existência da discreta diversidade de grau de escolaridade evidente entre os estudos, como provenientes das desigualdades socioeconômicas presentes nas distintas regiões brasileiras, que possivelmente proporcionam maior dificuldade para obtenção oportunidade de acesso à educação. Geralmente, o nível de instrução é fundamental para um bom desenvolvimento social, político e econômico de todo cidadão, todavia quando não é estabelecido de forma adequada pode influenciar em várias situações, inclusive a saúde, predispondo assim, este perfil de idoso a ter menos chances de possuir acesso a saúde de qualidade e a ter poucas condições financeiras, ocasionando uma maior probabilidade de agravos em saúde ${ }^{17,18}$.

Quanto a religião, a maioria dos idosos autorreferiram ser católicos (69,3\%). Conforme o IBGE9 a maior parte da população brasileira é cristã e de filiação católica. A religião para o idoso pode contribuir como suporte no enfrentamento e na superação do cotidiano, das perdas e no luto, além de proporcionar segurança e o conforto espiritual ${ }^{19}$.

Com relação a situação conjugal, os participantes desta pesquisa eram viúvos (52,6\%), corroborando com os estudos de Fernandes et al. ${ }^{11}$ e Mendonça et al. ${ }^{13}$ que também apresentaram uma maior frequência idosos viúvos.

A prevalência de DCNT na população estudada foi de 116 (85,9\%) idosos afetados por uma diversidade de 14 comorbidades crônicas. Evidenciou-se que 14\% dos idosos referiram não possuir nenhuma morbidade.

O Quadro 1 mostra a distribuição das DCNT em relação ao sexo, aproximadamente $88 \%$ do sexo feminino apresenta 


\section{artigo}

Lobato, T.C.L.; Reis, D.A.; Dantas, J.S.; Souza, D.B.M.; Souza, A.D.; Siqueira, G.G.;

Prevalência de doenças crônicas não transmissiveis em idosos longevos em um município no interior do Amazonas

alguma doença crônica enquanto no sexo masculino esse dado representa $82,7 \%$ dos casos. Entre as doenças autorreferidas pelos idosos podemos destacar HAS $(69,6 \%)$, dorsalgia $(30,4 \%)$, artrite reumatoide $(23,7 \%)$ e diabetes mellitus (17,8\%).

Silva e Tomaz ${ }^{16}$ encontram resultados em sua pesquisa, na qual identificaram uma prevalência da HAS $(60,8 \%)$, diabetes mellitus $(28,3 \%)$, doenças reumáticas $(22,5 \%)$ e cardiovasculares (15,1\%); nesse pressuposto, a pesquisa ainda ressalta que a incidência específica dessas doenças está relacionada ao processo de envelhecimento do senário atual, no qual à uma maior tendência global ao desenvolvimento de comorbidades crônico degenerativas não transmissíveis em detrimento das doenças infecto contagiosas.

Com relação a particularidade da predominância neste estudo de algumas comorbidades relacionadas ao sistema esquelético podemos inferir que esses resultados possuem relação com as atividades laborais exercidas pelos idosos ao longo de suas vidas, na região amazônica trabalhar com agricultura e pesca faz parte de uma herança cultural, sendo atividades que exigem um enorme esforço físico, muitas vezes até os limites do corpo.

Tabela 2. Associação entre morbidade autorreferida e sexo dos idosos longevos (N=135). Coari, AM, 2020.

\section{MORBIDADE}

\section{FEMININO}

$\mathrm{N}(\%)$

MASCULINO

$\mathbf{N}(\%)$

P*

\section{Hipertensão Arterial Sistêmica}

Não

Sim

23(27,7)

$60(72,3)$

$34(65,4)$

Doença na Coluna

Não

$60(72,3)$

Sim

$23(27,7)$

$34(65,4)$

$18(34,6)$

0,396

Artrite reumatoide

Não

$64(77,1)$

$39(75,0)$

Sim

19(22,9)

$13(25,0)$

0,779

Diabetes Mellitus

Não

$65(78,3)$

18(21,7)

46(88,5)

Sim

68(81,9)

$15(18,1)$

$50(96,2)$

Não

Sim

Demência

Não

Sim

$73(88,0)$

10(12,0)

46(88,5)

6(11,5)

0,929

\section{Alzheimer}

Não

80(96,4)

$3(3,6)$

$51(98,1)$

Sim

Gastrite crônica

Não

$14(16,8)$

$14(16,8)$

$47(90,4)$

Sim

Nota: *Teste Qui-quadrado; **Teste Exato de Fisher.
Um estudo realizado em uma cidade do interior da Bahia apresentou sete comorbidades referidas pelos idosos, as quais foram: depressão, diabetes, cardiopatia, doenças renais, câncer e hipertensão arterial sistêmica, sendo que, a hipertensão e o diabetes apresentaram os maiores índice dentre todas ${ }^{20}$. Em um município de Porto Alegre (RS) uma pesquisa $^{5}$ fez um levantamento das doenças crônicas autorreferidas e a sua associação com os sintomas de depressão, constatou que uma elevada prevalência de DCNT $(81,3 \%)$ entre os participantes, sobretudo de doenças como HAS $(70,8 \%)$ e DM (27,0\%), e posteriormente os distúrbios cardiovasculares.

Com relação a análise da associação entre a morbidade autorreferida e o sexo dos idosos longevos é apresentada na Tabela 2. Constou-se que apresentou associação estatística entre a morbidade osteoporose com o sexo.

Importa mencionar que ainda são escassos estudos com os longevos no Brasil, especificamente na região Norte. $\mathrm{O}$ que faz a importância dos achados encontrados na pesquisa. Espera-se que novas investigações científicas possam ser desenvolvidas com o aprofundamento do tema sobre DCNT em idoso longevo no contexto Amazônico, além de preencher uma lacuna de pesquisa neste cenário.

Com relação as limitações do estudo, considera-se que o desenho da pesquisa não permite inferir relação causal e efeito entre as variáveis estudadas. Outra questão de possíveis víeis da pesquisa é a própria memória do participante do estudo, que devido ao processo natural do envelhecimento pode se encontrar debilitada.

\section{CONCLUSÃO}

O estudo permitiu identificar as Doença Crônica Não Transmissíveis autorreferidas mais prevalentes, constatando-se que HAS, doenças na coluna, artrite reumatoide e a Diabetes Mellitus possuem maior incidência nos idosos longevos estudados. Assim como caracterizar o perfil sociodemográfico de idosos inscritos em unidades 
de saúde, em que sua maioria possui uma média de idade de 85,8 anos, sendo mulheres, viúvas, analfabetas e aposentadas.
Desse modo, os resultados contribuem de maneira propositiva para subsidiar planejamento de estratégia de Intervenções para equipe da Estratégia Saúde da Família, principalmente na promoção e prevenção ao idoso longevo.

\section{REFERENCES}

1. Belasco AGS, Okuno MFP. Reality and challenges of ageing. Rev Bras Enferm. 2019;72(Suppl 2):1-2. doi: http://dx.doi. org/10.1590/0034-7167.2019-72suppl201

2. Ribeiro DR, Calixto DM, Da Silva LL, Alves RPCN, Souza LMC. Prevalência de diabetes mellitus e hipertensão em idosos. Artigos@ [Internet]. 28 jan. 2020 [acesso em 24 jun.2020];14:e2132. Disponivel em: https://acervomais.com.br/index.php/artigos/article/view/2132

3. Gavasso WC, Beltrame V. Capacidade funcional e morbidades relatadas: análise comparativa em idosos. Rev. bras. geriatr. gerontol. [Internet]. 2017 [acesso em 15 ago. 2020]; 20(3): 398408. Disponivel em: http://www.scielo.br/scielo.php?script=sci_ arttext\&pid=S1809-98232017000300398\&lng=en

4. Simão L, Lages L, Paiva M, Ribeiro N, Araújo E, Leão G. Perfil dos idosos com doenças crônicas não transmissiveis internados em unidade de terapia intensiva. Enfermagem em Foco. 2019; 10(1). doi: https://doi.org/10.21675/2357-707X.2019.v10.n1.1329

5. Silva AR, Sgnaolin V, Nogueira EL, Loureiro F, Engroff $P$, Gomes I. Doenças crônicas não transmissiveis e fatores sociodemográficos associados a sintomas de depressão em idosos. J. bras. psiquiatr. Mar. 2017; 66(1): 45-51. doi: https://doi.org/10.1590/00472085000000149

6. Melo LA, Braga LC, Leite FPP, Bittar BF, Oséas JMF, Lima KC. Fatores associados à multimorbidade em idosos: uma revisão integrativa da literatura. Rev. bras. geriatr. gerontol. [Internet]. 2019 [acesso em 15 ago. 2020]; 22(1): e180154. Disponivel em: http://www.scielo.br/scielo.php?script=sci_arttext\&pid=S1809-98232019000100302\&lng=en

7. Cavalcanti G, Doring M, Portella MR, Bortoluzzi EC, Mascarelo A, Dellani MP. Multimorbidity associated with polypharmacy and negative self-perception of health. Rev. bras. geriatr. gerontol. [Internet]. 2017 [acesso em 15 ago. 2020]; 20(5): 634-642. Disponivel em: http://www.scielo.br/scielo.php?script=sci_arttext\&pid=S1809-98232017000500634\&lng=en

8. Cruz PKR, Vieira MA, Carneiro JA, Costa FM, Caldeira AP. Difficulties of access to health services among non-institutionalized older adults: prevalence and associated factors. Rev. bras. geriatr. gerontol. [Internet]. 2020 [acesso em 15 ago. 2020]; 23(6): e190113. Disponivel em: http://www.scielo.br/scielo.php?script=sci_arttext\&pid=S1809-98232020000600201\&lng=en

9. IBGE. Instituto Brasileiro de Geografia e Estatística. Censo Demográfico 2010- Características gerais da população, religião e pessoas com deficiência. 2012; 1:1-30.

10. Llano PMP, Lange C, Sequeira CAC, Jardim VMR, Castro DSP, Santos F. Factors associated with frailty syndrome in the rural elderly. Rev Bras Enferm. 2019;72(Suppl 2):14-21. doi: http://dx.doi. org/10.1590/0034-7167-2017-0079

11. Fernandes DS, Gonçalves LHT, Ferreira AMR, Santos MIPO.
Avaliação da capacidade funcional de idosos longevos amazônidas. Rev. Bras. Enferm. 2019; 72(Suppl 2): 49-55. doi: http://dx.doi. org/10.1590/0034-7167-2017-0798

12. Tavares DMS, Gomes NC, Soares LAL, Marchiori GF. Fatores associados à independência funcional de idosos longevos da comunidade. Cogitare enferm [Internet]. 2019 [acesso em 10 jul. 2020]; e61527. Disponivel em: https://revistas.ufpr.br/cogitare/ article/view/61527

13. Mendonça SS, Marques APOM, Nunes MGS, D'Angelo ER, Leal MCC. Capacidade funcional de idosos longevos: análise transversal baseada em um modelo de decisão. Geriatr Gerontol Aging [Internet]. 2020 [acesso em 5 jul. 2020];14(1):52-60. Disponivel em: https://cdn.publisher.gn1.link/ggaging.com/pdf/v14n1a09.pdf

14. Hatice Simsek, Erdem Erkoyun, Ali Akoz, Alp Ergor, Reyhan Ucku. Falls, fear of falling and related factors in community dwelling individuals aged 80 and over in Turkey. Australasian Journal on Aging. 2020; 39(1): e16-e23. doi: https://doi-org.ez2.periodicos. capes.gov.br/10.1111/ajag.12673

15. Joelle $\mathrm{H}$. Fong. Disability incidence and functional decline among older adults with major chronic diseases. BMC Geriatrics. 2019; 19(1): 1-9. doi: https://doi.org/10.1186/s12877-019$1348-z$

16. Silva MVM, Tomaz AF. Análise da qualidade de vida e capacidade funcional de idosos. revista on-line do cesed - centro de ensino superior e desenvolvimento. [Internet]. 2017 [acesso 23 jul. 2020]; 18(28/29). Disponível em: http://revistatema.facisa.edu.br/ index.php/revistatema/article/view/1015/pdf

17. Machado WD, Gomes DF, Freitas CASL, Brito MCC, Moreira ACA. (Elderly with not transmitted chronic diseases: a group association study). ReonFacema. [Internet]. 2017 [acesso em 23 jun. 2020]; 3(2), 444-451. Disponivel em: http://www.facema.edu.br/ ojs/index.php/ReOnFacema/article/download/194/106

18. Billett MC, Campanharo CRV, Lopes MCBT, Batista REA, Belasco AGS, Okuno MFP. Capacidade funcional e qualidade de vida de octogenários hospitalizados. Rev. Bras. Enferm. 2019; 72(Suppl 2): 43-48. doi: https://doi.org/10.1590/0034-7167-2017-0781.

19. Nunes MGS, Leal MCC, Marques APO, Mendonça SS. Idosos longevos: avaliação da qualidade de vida no domínio da espiritualidade, da religiosidade e de crenças pessoais. Saúde debate [Internet]. 2017 [acesso em 10 jul. 2020]; 41 (115): 1102-1115. Disponivel em: https://www.scielo.br/scielo.php?pid=S0103-110 42017000401102\&script=sci_abstract\&tlng=pt

20. Abreu SSS, Oliveira AG, Macedo MASS, Duarte SFP, Reis LA, Lima PV. Prevalência de Doenças Crônicas não Transmissíveis em Idosos de uma Cidade do Interior da Bahia. Id on Line Revista Multidisciplinar e de Psicologia [Internet]. 2017;11(38):65262. Disponivel em: https://idonline.emnuvens.com.br/id/article/ view/963/1367 